\title{
Isolated Hypomethylation of IGF2 Associated with Severe Hypoglycemia Responsive to Growth Hormone Treatment
}

\author{
Sarah C. Grünert*(D), Uta Matysiak, Franka Hodde, Gunda Ruzaike, Ekkehart Lausch, Anke Schumann, \\ Natascha van der Werf-Grohmann, Ute Spiekerkoetter and Miriam Schmidts
}

Citation: Grünert, S.C.; Matysiak, U.; Hodde, F.; Ruzaike, G.; Lausch, E.; Schumann, A.; van der WerfGrohmann, N.; Spiekerkoetter, U.; Schmidts, M. Isolated

Hypomethylation of IGF2 Associated with Severe Hypoglycemia Responsive to Growth Hormone Treatment. Diagnostics 2021, 11, 749. https://doi.org/10.3390/ diagnostics 11050749

Academic Editor: Consolato M. Sergi

Received: 26 March 2021

Accepted: 16 April 2021

Published: 22 April 2021

Publisher's Note: MDPI stays neutral with regard to jurisdictional claims in published maps and institutional affiliations.

Copyright: (C) 2021 by the authors Licensee MDPI, Basel, Switzerland. This article is an open access article distributed under the terms and conditions of the Creative Commons Attribution (CC BY) license (https:// creativecommons.org/licenses/by/ $4.0 /)$.
Department of General Paediatrics, Adolescent Medicine and Neonatology, Faculty of Medicine, Medical Centre-University of Freiburg, 79106 Freiburg, Germany; uta.matysiak@uniklinik-freiburg.de (U.M.); franka.hodde@uniklinik-freiburg.de (F.H.); gunda.ruzaike@uniklinik-freiburg.de (G.R.); ekkehart.lausch@uniklinik-freiburg.de (E.L.); anke.schumann@uniklinik-freiburg.de (A.S.); natascha.vdwerf-grohmann@uniklinik-freiburg.de (N.v.d.W.-G.); ute.spiekerkoetter@uniklinik-freiburg.de (U.S.); miriam.schmidts@uniklinik-freiburg.de (M.S.)

* Correspondence: sarah.gruenert@uniklinik-freiburg.de; Tel.: +49-761-270-43000

\begin{abstract}
Hypomethylation of H19 and IGF2 can cause Silver-Russell syndrome (SRS), a clinically and genetically heterogeneous condition characterized by intrauterine growth restriction, poor postnatal growth, relative macrocephaly, craniofacial abnormalities, body asymmetry, hypoglycemia and feeding difficulties. Isolated hypomethylation of IGF2 has been reported in single cases of SRS as well. Here, we report on a 19-month-old patient who presented with two episodes of hypoglycemic seizures. No intrauterine growth restriction was observed, the patient did not present with SRS-typical facial features, and postnatal growth in the first months of life was along the lower normal percentiles. Exome sequencing did not reveal any likely pathogenic variants explaining the phenotype; however, hypomethylation studies revealed isolated hypomethylation of IGF2, while the methylation of H19 appeared normal. Hypoglycemia responded well to growth hormone therapy, and the boy showed good catch-up growth. Our case demonstrates that SRS and isolated IGF2 hypomethylation should be considered early in the diagnosis of recurrent hypoglycemia in childhood, especially in combination with small gestational age and poor growth.
\end{abstract}

Keywords: IGF2; Silver-Russel syndrome; hypomethylation; hypoglycemia; growth hormone

\section{Introduction}

Hypomethylation of the imprinted telomeric control region 1 (ICR1) at 11p15.5 on the paternal allele is the most common genetically confirmed cause of Silver-Russel syndrome (SRS, OIMIM \#180860), accounting for 35-50\% of all cases [1-5]. Two genes lie within this region: H19 and IGF2 (insulin-like growth factor 2). Their expression is controlled by an intergenic differentially methylated region, DMR1, where an insulator between H19 and IGF2 inhibits H19 enhancer effects on IGF2 so that IGF2 is not expressed. ICR1 methylation occurring only on the paternal allele abrogates the insulator effects so that the H19 enhancer is able to activate IGF2 expression. ICR1 hypomethylation on the paternal allele can therefore lower IGF2 expression, resulting in the clinical picture of SRS [6,7]. For SRS cases, methylation studies often reveal hypomethylation of H19 and IGF2, while only very few cases presenting with phenotypic characteristics overlapping SRS with isolated H19 or IGF2 hypomethylation have been reported to date [7]. Likewise, pathogenic paternal IGF2 variants have been identified in SRS patients [8]. Rarely, SRS is caused by maternal uniparental disomy of chromosome 7 (mUPD7; 7-10\% of SRS cases [9-11]), duplications, deletions, or translocations involving chromosome 7 and by pathogenic variants in CDKN1C (maternal allele) [12], PLAG1 [13], and HMGA2 [14]. Interestingly, about $40 \%$ of individuals who meet clinical criteria for SRS have negative molecular and/or cytogenetic testing [6]. 
SRS is characterized by severe asymmetric intrauterine growth retardation (IUGR), resulting in affected individuals being born small for gestational age (SGA) with relative macrocephaly at birth. Additional clinical features include craniofacial abnormalities such as a prominent forehead usually with frontal bossing and a triangular facies, fifth-finger clinodactyly, micrognathia with narrow chin, body asymmetry, and a variety of minor malformations [6]. Postnatal growth is also impaired, and the average adult height in untreated individuals is $\sim 3.1 \pm 1.4 \mathrm{SD}$ below the mean [6]. Some patients with this condition show developmental delay (both motor and cognitive) and learning disabilities [6,15]. Children are at risk for fasting hypoglycemia, which can be explained by little subcutaneous fat, reduced body mass, feeding difficulties and poor appetite, and - in several children-by growth hormone (GH) deficiency [16].

Several clinical scoring systems for the diagnosis of SRS have been suggested $[3,7,17,18]$, with the majority of them requiring the presence of three or four out of the following characteristic features: birth weight $\leq-2 \mathrm{SD}$, postnatal growth restriction, relative macrocephaly, facial characteristics and body asymmetry. The Netchine-Harbison Clinical Scoring System (NH-CSS), published in 2015, adds feeding difficulties as a sixth classical feature and requires four out of six criteria for the diagnosis of "likely SRS" [17].

Here, we report on a 19-month-old patient who presented with recurrent severe and symptomatic hypoglycemia. While he did not meet SRS clinical diagnostics criteria, we detected the isolated hypomethylation of IGF2. Although GH deficiency could not be demonstrated from peripheral blood samples, blood glucose concentrations stabilized under growth hormone treatment.

\section{Methods}

\subsection{Genetic Diagnostics}

Written consent was obtained from the patient's parents.

\subsection{Exome Sequencing}

Exome sequencing and data analysis were performed as previously described [19,20]. In brief, DNA was extracted from $5 \mathrm{~mL}$ of ethylenediamine tetraacetic acid (EDTA) blood using a commercial kit (DNAeasy blood and tissue kit, Quiagen, Germantown, MD20874, USA). Two micrograms of DNA was used for library preparation and exome sequencing performed using Agilent sure select V6 kit. Variant filtering was performed for variants with a minor allele frequency below $1 \%$ in public databases such as gnomAD, 1000 genomes and ExAc as well as for coding variants and variants within $8 \mathrm{bp}$ distance of splice sites.

\subsection{Methylation-Specific Multiplex Ligation-Dependent Probe Amplification (MS-MLPA)}

To investigate methylation within the IC2 (KvDMR) and IC1 (H19DMR) domains in the 11p15 BWS/RSS region, MS-MLPA was performed using SALSAMLPA probemix ME030 BWS; Russel Silver Syndrome (RSS) according the manufacturer's protocol (MRC Holland, Amsterdam, The Netherlands). The kit contains 26 probes specific for the BWS/RSS 11p15 region with ten of these probes containing an HhaI recognition site and providing information on the methylation status of the BWS/RSS 11p15 region.

\section{Case Presentation}

The patient is the second child of non-consanguineous German parents. The fouryear-old sister is healthy, but of short stature ( $<3$ rd centile at age 4 years). The mother suffered from gestational diabetes during both pregnancies, but otherwise no metabolic or endocrinological disorders were reported within the family. The patient was born at $40+2$ weeks of gestation with a birth weight of $3100 \mathrm{~g}$ (11th centile) and a body length of $50 \mathrm{~cm}$ (12th centile). The boy was first presented to our metabolic clinic at the age of 19 months with a history of two hypoglycemic seizures within 2 weeks. Both events occurred after he had slept through the night for the first times. When the mother tried to wake him up in the morning, he was apathic and subsequently showed convulsions 
of his upper body. The parents gave him apple puree which led to rapid cessation of the seizure. While waiting for the emergency doctor, he ate yoghurt and sausage. Upon the arrival of the emergency team, all clinical symptoms had subsided, and the blood sugar was $4.4 \mathrm{mmol} / \mathrm{L}$. A second very similar episode occurred 2 weeks later. The parents further reported that the boy used to wake up in the night demanding food.

Until the age of 19 months, he was normally developed, and no hypoglycemiasuggestive symptoms were reported. His growth was always within the lower normal range (see Figure 1).

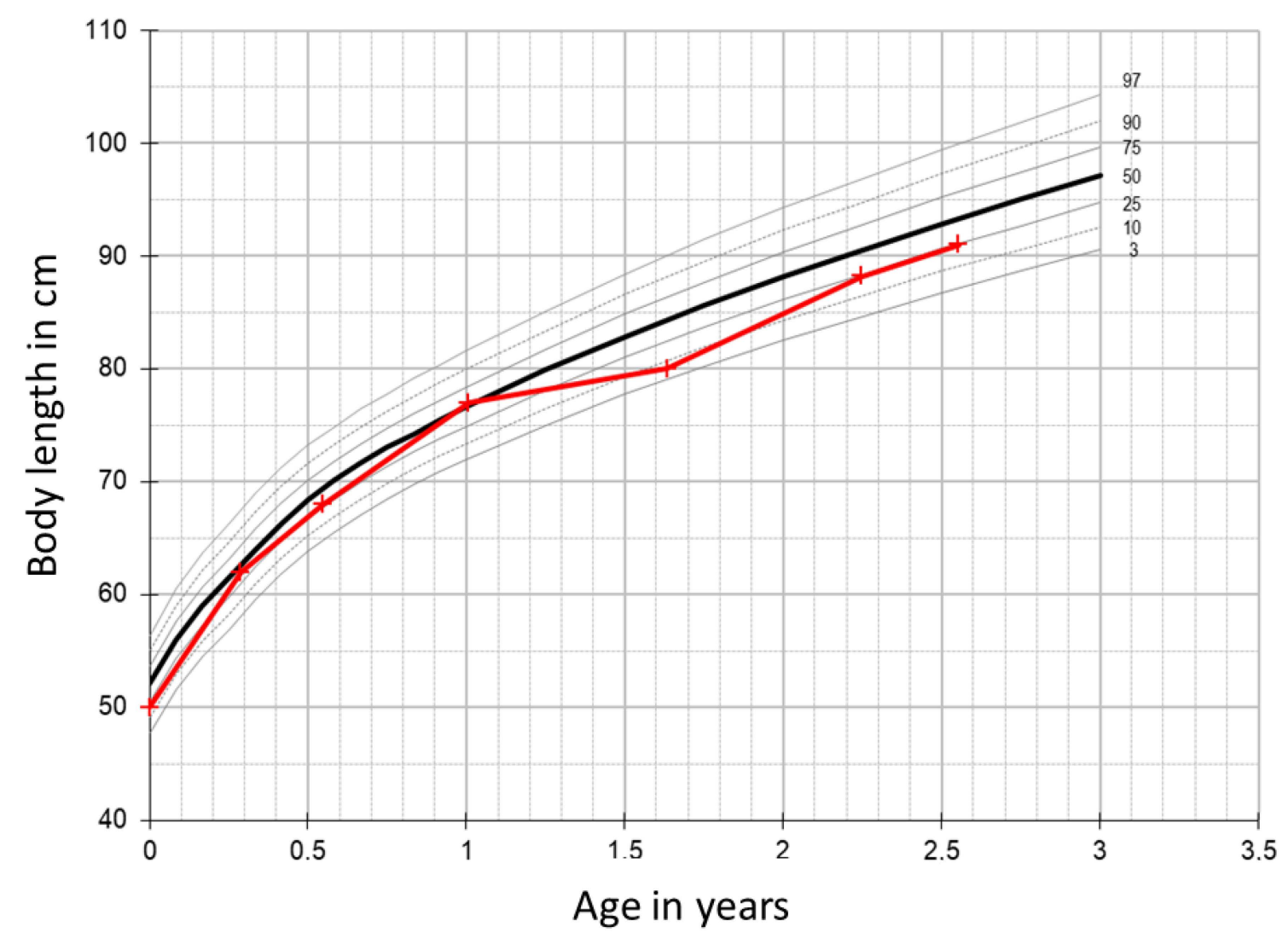

Figure 1. Growth chart of the patient. The boy was not small for gestational age, and his further growth was within the lower normal range.

His appetite was poor, but no special eating habits were reported. No febrile or gastrointestinal infections had occurred in the past. The local pediatrician recommended measuring the blood glucose concentration before breakfast for one week. This revealed two severe asymptomatic hypoglycemias of $2.1 \mathrm{mmol} / \mathrm{L}$, respectively.

At presentation in the outpatient clinic, his clinical condition was very good. His body weight was $10.5 \mathrm{~kg}$ (15th centile) and his body length was $80 \mathrm{~cm}$ (6th centile) (Figure 1). The clinical examination was unremarkable. Laboratory work-up including metabolic investigations (urinary organic acids, acylcarnitines in dried blood spots, amino acids in serum) yielded normal results. Serum triglycerides $(126 \mathrm{mg} / \mathrm{dL}$, nor$\mathrm{mal}<150 \mathrm{mg} / \mathrm{dL}$ ), total cholesterol (134 mg/dL, normal 50-200 mg/dL) and lactic acid $(1.5 \mathrm{mmol} / \mathrm{L}$, normal $<1.6 \mathrm{mmol} / \mathrm{L})$ were normal. Serum IGF1 and IGFBP3 levels were also within the reference range. GH deficiency was excluded by an arginine stimulation test (30 min after arginine supplementation growth hormone concentration $11.3 \mathrm{ng} / \mathrm{mL}$; normal $>8.0 \mathrm{ng} / \mathrm{mL}$ ). An oral glucose tolerance test was performed and no signs of impaired glucose tolerance or insulin resistance were observed. Abdominal sonography was also normal. Further diagnostic work-up including whole exome sequencing and methylation studies for SRS was initiated. Until the results became available, symptomatic 
treatment with uncooked cornstarch at bedtime and during the night was started and no further symptomatic hypoglycemic events were observed; however, continuous glucose monitoring with a Freestyle libre ${ }^{\circledR} 2$ device (subcutaneous glucose sensor by Abbot) was implemented and confirmed recurrent, severe but asymptomatic hypoglycemia.

Whole exome sequencing yielded no likely pathogenic variants explaining the phenotype. Methylation studies (MLPA) of the region 11p15, the critical methylation-sensitive region in patients with SRS, yielded normal results for four H19DMR probes, but the signal of the IGF2 probe was decreased to $50 \%$ compared to healthy controls, suggesting hypomethylation of the paternally expressed IGF2 gene (Figure 2).

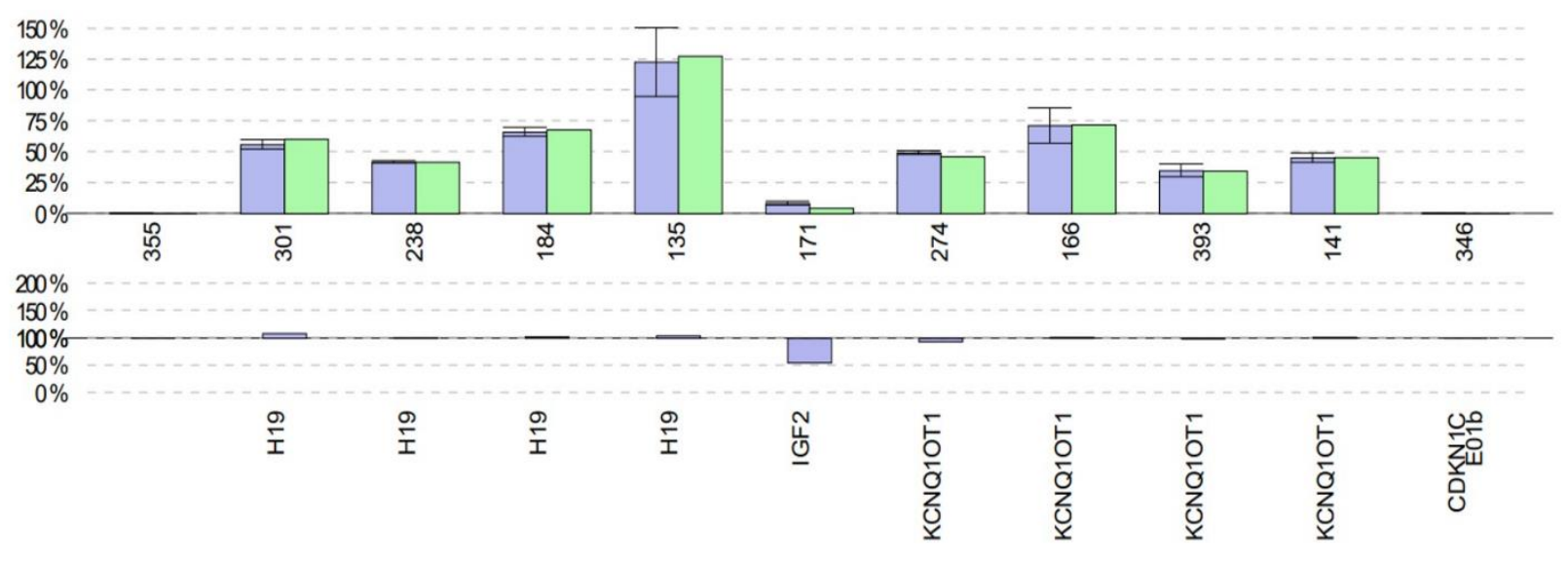

Figure 2. IGF2 hypomethylation revealed by H19DMR MLPA. While no difference between patient (green) and controls (purple) (upper panel) was observed for four H19, four KCNQ1OT1 and one CDKN1C probe, IGF2 methylation was 50\% lower in the patient compared to the controls (lower panel, purple).

GH therapy was therefore initiated at the age of 2 years and led to significant stabilization of glucose homeostasis. Cornstarch supplementation could be stopped, and no further severe hypoglycemias have occurred so far. The boy showed a good catch-up growth of 4 $\mathrm{cm}$ within the first 3 months of GH treatment.

\section{Discussion}

We report on a patient with IGF2 hypomethylation who presented with severe recurrent hypoglycemia at the age of 19 months. The $11 \mathrm{p} 15$ region comprises two imprinted domains that are important for the control of fetal and postnatal growth, ICR1 and ICR2 [7]. ICR1 coordinates the expression of two oppositely imprinted genes, H19 and IGF2. A study by Bartholdi et al. demonstrated that the majority of SRS patients with methylation abnormalities show hypomethylation at both the H19 and IGF2 genes [7]. Only a small subset of patients carries epimutations restricted to either the H19 or IGF2 gene as seen in our patient, in whom only hypomethylation of IGF2 was detected [7]. Due to the low number of reported cases, it is not clear if the phenotypes in cases with isolated IGF2 hypomethylation and cases with hypomethylation of the entire region are comparable. Importantly, our patient did not meet SRS clinical diagnosis criteria (no IUGR/small for gestational age, low-normal postnatal growth, no typical craniofacial features, and no body asymmetries). We performed MLPA analyses to rule out SRS as a cause of the severe hypoglycemic episodes.

It is well known that children with SRS are prone to hypoglycemia, especially after prolonged fasting [6,21]. The precise mechanism of hypoglycemia in SRS and likewise IGF2 dysfunction is not clear to date, but several factors are discussed: IGF2 presumably is able to bind to both IGF1 and IGF2 receptors as well as the insulin receptor and interacts with IGFBP3, possibly influencing glucose homeostasis [16]. It is further assumed that lower GH levels in SRS may contribute to a reduced fasting tolerance in SRS. Young children with SRS have little subcutaneous fat, low muscle and liver mass, and a disproportionately 
large brain-for-body size [16,21]. Additionally, many patients have poor appetite, oral motor problems, and feeding disorders [22]. GH deficiency has been described in several cases [16]. The incidence of hypoglycemia is approximately $27 \%$ [15], with a high frequency of spontaneous, asymptomatic nocturnal hypoglycemia [16,21]. Although hypoglycemia is quite common in SRS patients, many remain asymptomatic; therefore, hypoglycemic seizures as observed in our patient are usually not the first presenting symptom. It can be assumed that our patient had recurrent asymptomatic hypoglycemia before he finally presented with a hypoglycemic seizure. In several studies, good response to GH treatment in SRS children with respect to growth was shown [23,24]. The goals of GH treatment, however, are not only to improve growth velocity but also to improve body composition, psychomotor development, and appetite, to optimize linear growth, to increase lean body mass and muscle power and to reduce the risk of hypoglycemia [21]. In our patient, GH treatment was mainly started to better control hypoglycemia. Indeed, GH therapy resulted in a marked improvement of glucose homeostasis.

\section{Conclusions}

Our case demonstrates that severe hypoglycemia may be the first presenting symptom for SRS-like cases associated with IGF2 hypomethylation. SRS and IGF2 hypomethylation should be considered early in the differential diagnosis of recurrent ketotic hypoglycemia in childhood, as patients benefit from early GH treatment.

Author Contributions: S.C.G. was responsible for the clinical management of the patient and drafted the manuscript together with M.S., U.M., G.R., M.S. and E.L. were responsible for the molecular diagnostics including methylation studies, and U.M. drafted Figure 2. F.H., A.S., N.v.d.W.-G., and U.S. were involved in the clinical diagnostics and treatment of the patient. All authors have read and agreed to the published version of the manuscript.

Funding: M.S. acknowledges funding from the European Research Council (ERC) (ERC starting grant TREATCilia, grant agreement 716344), and M.S. and E.L. received funding from the Deutsche Forschungsgemeinschaft (DFG, German Research Foundation)—Project-ID 431984000—SFB 1453.

Institutional Review Board Statement: Ethics approval and consent to participate: Not applicable.

Informed Consent Statement: The patient's parents gave their informed consent for publication.

Data Availability Statement: Not applicable.

Acknowledgments: We are grateful to the family for their support of this work. This work was supported by the Metabolic Division in the University Children's Hospital, which is part of the Freiburg Center for Rare Diseases. Several authors of this publication are members of the European Reference Network for Rare Hereditary Metabolic Disorders (MetabERN)-Project ID No 739543. The article processing charge was funded by the Baden-Wuerttemberg Ministry of Science, Research and Art and the University of Freiburg in the funding program Open Access Publishing.

Conflicts of Interest: The authors declare that they have no competing interests.

$\begin{array}{ll}\text { Abbreviations } \\ \text { GH } & \text { Growth hormone } \\ \text { IUGR } & \text { Intrauterine growth restriction } \\ \text { NH-CSS } & \text { Netchine-Harbison Clinical Scoring System } \\ \text { SGA } & \text { Small for gestational age } \\ \text { SRS } & \text { Silver-Russell syndrome }\end{array}$

\section{References}

1. Bliek, J.; Terhal, P.; van den Bogaard, M.-J.; Maas, S.; Hamel, B.; Salieb-Beugelaar, G. Hypomethylation of the H19 gene causes not only Silver-Russell syndrome (SRS) but also isolated asymmetry or an SRS-like phenotype. Am. J. Hum. Genet. 2006, 78, 604-614. [CrossRef]

2. $\quad$ Eggermann, T.; Schönherr, N.; Meyer, E.; Obermann, C.; Mavany, M.; Eggermann, K. Epigenetic mutations in 11p15 in SilverRussell syndrome are restricted to the telomeric imprinting domain. J. Med. Genet. 2006, 43, 615-616. [CrossRef] 
3. Netchine, I.; Rossignol, S.; Dufourg, M.-N.; Azzi, S.; Rousseau, A.; Perin, L. 11p15 imprinting center region 1 loss of methylation is a common and specific cause of typical Russell-Silver syndrome: Clinical scoring system and epigenetic-phenotypic correlations. J. Clin. Endocrinol. Metab. 2007, 92, 3148-3154. [CrossRef]

4. Yamazawa, K.; Kagami, M.; Nagai, T.; Kondoh, T.; Onigata, K.; Maeyama, K. Molecular and clinical findings and their correlations in Silver-Russell syndrome: Implications for a positive role of IGF2 in growth determination and differential imprinting regulation of the IGF2-H19 domain in bodies and placentas. J. Mol. Med. 2008, 86, 1171-1181. [CrossRef] [PubMed]

5. Gicquel, C.; Rossignol, S.; Cabrol, S.; Houang, M.; Steunou, V.; Barbu, V. Epimutation of the telomeric imprinting center region on chromosome 11p15 in Silver-Russell syndrome. Nat. Genet. 2005, 37, 1003-1007. [CrossRef]

6. Saal, H.M.; Harbison, M.D.; Netchine, I. Silver-Russell Syndrome GeneReviews ${ }^{\circledR}$; Adam, M.P., Ardinger, H.H., Pagon, R.A., Wallace, S.E., Bean, L.J., Stephens, K., Eds.; University of Washington: Seattle, WA, USA, 1993. Available online: http://www. ncbi.nlm.nih.gov/books/NBK1324/ (accessed on 6 June 2020).

7. Bartholdi, D.; Krajewska-Walasek, M.; Ounap, K.; Gaspar, H.; Chrzanowska, K.H.; Ilyana, H. Epigenetic mutations of the imprinted IGF2-H19 domain in Silver-Russell syndrome (SRS): Results from a large cohort of patients with SRS and SRS-like phenotypes. J. Med. Genet. 2009, 46, 192-197. [CrossRef] [PubMed]

8. Begemann, M.; Zirn, B.; Santen, G.; Wirthgen, E.; Soellner, L.; Büttel, H.-M. Paternally Inherited IGF2 Mutation and Growth Restriction. N. Engl. J. Med. 2015, 373, 349-356. [CrossRef] [PubMed]

9. Bernard, L.E.; Peñaherrera, M.S.; Van Allen, M.I.; Wang, M.S.; Yong, S.L.; Gareis, F. Clinical and molecular findings in two patients with russell-silver syndrome and UPD7: Comparison with non-UPD7 cases. Am. J. Med. Genet. 1999, 87, 230-236. [CrossRef]

10. Price, S.M.; Stanhope, R.; Garrett, C.; Preece, M.A.; Trembath, R.C. The spectrum of Silver-Russell syndrome: A clinical and molecular genetic study and new diagnostic criteria. J. Med. Genet. 1999, 36, 837-842.

11. Eggermann, T.; Schönherr, N.; Jäger, S.; Spaich, C.; Ranke, M.B.; Wollmann, H.A. Segmental maternal UPD(7q) in Silver-Russell syndrome. Clin. Genet. 2008, 74, 486-489. [CrossRef]

12. Binder, G.; Ziegler, J.; Schweizer, R.; Habhab, W.; Haack, T.B.; Heinrich, T. Novel mutation points to a hot spot in CDKN1C causing Silver-Russell syndrome. Clin. Epigenetics 2020, 12, 152. [CrossRef]

13. Abi Habib, W.; Brioude, F.; Edouard, T.; Bennett, J.T.; Lienhardt-Roussie, A.; Tixier, F. Genetic disruption of the oncogenic HMGA2-PLAG1-IGF2 pathway causes fetal growth restriction. Genet. Med. 2018, 20, 250-258. [CrossRef]

14. Inoue, T.; Nakamura, A.; Iwahashi-Odano, M.; Tanase-Nakao, K.; Matsubara, K.; Nishioka, J. Contribution of gene mutations to Silver-Russell syndrome phenotype: Multigene sequencing analysis in 92 etiology-unknown patients. Clin. Epigenetics 2020, 2, 86. [CrossRef] [PubMed]

15. Wakeling, E.L.; Amero, S.A.; Alders, M.; Bliek, J.; Forsythe, E.; Kumar, S. Epigenotype-phenotype correlations in Silver-Russell syndrome. J. Med. Genet. 2010, 47, 760-768. [CrossRef]

16. Azcona, C.; Stanhope, R. Hypoglycemia and Russell-Silver syndrome. J. Pediatr. Endocrinol. Metab. 2005, 18, 663-670. [CrossRef]

17. Azzi, S.; Salem, J.; Thibaud, N.; Chantot-Bastaraud, S.; Lieber, E.; Netchine, I. A prospective study validating a clinical scoring system and demonstrating phenotypical-genotypical correlations in Silver-Russell syndrome. J. Med. Genet. 2015, 52, 446-453. [CrossRef] [PubMed]

18. Ishida, M. New developments in Silver-Russell syndrome and implications for clinical practice. Epigenomics 2016, 8, 563-580. [CrossRef]

19. Rad, A.; Altunoglu, U.; Miller, R.; Maroofian, R.; James, K.N.; Çağlayan, A.O. MAB21L1 loss of function causes a syndromic neurodevelopmental disorder with distinctive cerebellar, ocular, craniofacial and genital features (COFG syndrome). J. Med. Genet. 2019, 56, 332-339. [CrossRef]

20. Rehman, A.U.; Najafi, M.; Kambouris, M.; Al-Gazali, L.; Makrythanasis, P.; Rad, A. Biallelic loss of function variants in PPP1R21 cause a neurodevelopmental syndrome with impaired endocytic function. Hum. Mutat. 2019, 40, 267-280. [CrossRef]

21. Wakeling, E.L.; Brioude, F.; Lokulo-Sodipe, O.; O'Connell, S.M.; Salem, J.; Bliek, J. Diagnosis and management of Silver-Russell syndrome: First international consensus statement. Nat. Rev. Endocrinol. 2017, 13, 105-124. [CrossRef] [PubMed]

22. Fuke, T.; Mizuno, S.; Nagai, T.; Hasegawa, T.; Horikawa, R.; Miyoshi, Y. Molecular and clinical studies in 138 Japanese patients with Silver-Russell syndrome. PLoS ONE 2013, 8, e60105. [CrossRef] [PubMed]

23. Toumba, M.; Albanese, A.; Azcona, C.; Stanhope, R. Effect of long-term growth hormone treatment on final height of children with Russell-Silver syndrome. Horm. Res. Paediatr. 2010, 74, 212-217. [CrossRef] [PubMed]

24. Binder, G.; Liebl, M.; Woelfle, J.; Eggermann, T.; Blumenstock, G.; Schweizer, R. Adult height and epigenotype in children with Silver-Russell syndrome treated with GH. Horm. Res. Paediatr. 2013, 80, 193-200. [CrossRef] [PubMed] 